\title{
Epidemiologic and clinical characteristics of multisystem inflammatory syndrome in adults: a rapid review
}

\author{
Nicole Atchessi ${ }^{1}$, Rojiemiahd Edjoc ${ }^{1 *}$, Megan Striha ${ }^{1}$, Lisa Waddell ${ }^{2}$, Natalie Bresee ${ }^{3}$, \\ Thomas Dawson ${ }^{1}$
}

\begin{abstract}
Multisystem inflammatory disease in children (MIS-C) is one of the severe presentations of the coronavirus disease 2019 (COVID-19) that has been described in the literature since the beginning of the pandemic. Although MIS-C refers to children, cases with similar clinical characteristics have been recently described in adults. A description of the epidemiologic and clinical characteristics of multisystem inflammatory disease in adults (MIS-A) is a starting point for better knowledge and understanding of this emerging disease.

We identified nine case reports of MIS-A in the literature, five from the United States, two from France and two from the United Kingdom. The case descriptions revealed similarities in clinical features, including occurrence during post-acute disease phase, fever, digestive symptoms, cardiac involvement and elevated inflammatory markers. All the patients were hospitalized, three required admission to the intensive care unit and one died. The most common treatments were intravenous immunoglobulin, prednisolone and aspirin.
\end{abstract}

These findings suggest that MIS-A is a severe complication of COVID-19 disease that can lead to death. Further studies to improve our understanding of the pathogenesis of MIS-A, which will help improve treatment decisions and prevent sequelae or death.
This work is licensed under a Creative Commons Attribution 4.0 International License.

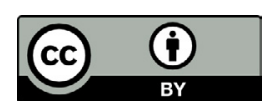

Affiliations

${ }^{1}$ Health Security Infrastructure Branch, Public Health Agency of Canada, Ottawa, ON

2 Public Health Risk Sciences Division, National Microbiology Laboratory, Public Health Agency of Canada, Guelph, ON

${ }^{3}$ Children's Hospital of Eastern Ontario, Ottawa, ON

*Correspondence:

rojiemiahd.edjoc@canada.ca

Suggested citation: Atchessi N, Edjoc R, Striha M, Waddell L, Bresee N, Dawson T. Epidemiologic and clinical characteristics of multisystem inflammatory syndrome in adults: a rapid review. Can Commun Dis Rep 2021;47(7/8):305-15. https://doi.org/10.14745/ccdr.v47i78a03

Keywords: COVID-19, SARS-CoV-2, MIS-A, MIS-C, multisystem inflammatory syndrome in adult

\section{Introduction}

The coronavirus disease 2019 (COVID-19) is a novel disease resulting from infection by severe acute respiratory syndrome coronavirus 2 (SARS-CoV-2) (1). As of May 29, 2021, the virus has infected more than 170 million people and caused more than 3.5 million deaths worldwide (2).

The clinical characteristics of COVID-19 disease vary from asymptomatic to severe. The most common symptoms are fever, cough, diarrhea and dyspnea (3). There are three clinical stages (4). The first stage is characterized by infection with SARS-CoV-2, with flu-like symptoms in certain cases. The second stage, characterized by viral pneumonia, possibly combined with pulmonary inflammation and coagulopathy, can require hospitalization and even mechanical ventilation. The third stage of the disease is characterized by fibrosis.
Multisystem inflammatory syndrome has been widely reported in children (5) and, more recently, in adults (6). In children, multisystem inflammatory syndrome (MIS-C) is a severe presentation that has been described in the literature since the beginning of the pandemic. Although MIS-C is defined as restricted to children, similar clinical characteristics have been described in adults. Knowing the epidemiologic and clinical characteristics of multisystem inflammatory syndrome cases in adults (MIS-A) provides a starting point to a better understanding of this emerging disease.

\section{Methods}

A database at the Public Health Agency of Canada is populated daily with new COVID-19 literature using standardized algorithms [e.g. "COVID-19" OR "SARS-CoV-2" OR "SARS-Coronoavirus-2" 
OR "nCov" OR "novel CoV" OR ("novel AND coronavirus")] tailored to each searched database, that is, PubMed, Scopus, BioRxiv, MedRxiv, ArXiv, SSRN and Research Square. The literature is cross-referenced with the COVID-19 information centres run by the Lancet, the BMJ, Elsevier and Wiley.

Our search through the Public Health Agency of Canada database included studies published in English since the start of the pandemic until November 13, 2020. We gathered details about COVID-19-related studies in a RefWorks database and an Excel spreadsheet that are searchable by topic. Search terms used to retrieve the MIS-A literature from titles and abstracts in the Excel spreadsheet included "MIS-A," "Kawasaki," "multisystem inflam*," "multi-system inflam*," "inflammatory multisystem," "inflammatory multi-system," "inflammatory disease," "Kawasaki-like" and "COVID-19 linked disease." We screened articles $(n=314)$ for relevance and included those that described MIS-A with a COVID-19 link (see Appendix A and Appendix B). We excluded paediatric cases and studies with cases similar to a MIS-A, but not formally diagnosed as MIS-A as per the authors. Since MIS-A is an emerging disease, a case definition does not yet exist. Authors of studies included in this review based case selection on the definition of MIS-C, while excluding the age criteria (see Appendix C).

\section{Results}

We identified nine case reports of MIS-A in the literature, five in the United States, two in France and two in the United Kingdom.

All nine cases of MIS-A occurred in relatively young adults, with a median age of 31 years (interquartile range [IOR] $=25-45$ years). Six patients were male (6-11). Six studies reported ethnicity: three patients were of African origin or African American $(6,8,12)$, two were of Hispanic origin $(11,13)$ and one was White $(10)$. Seven out of nine studies reported on comorbidity. Two patients had both hypertension and obesity $(6,12)$; one of these patients also had diabetes (12). Four patients had no known comorbidities $(7,8,10,13)$; in three cases the comorbidity status was not reported $(7,9,14)$.

All nine patients underwent a reverse transcription polymerase chain reaction (RT-PCR) test for COVID-19. Five had negative RT-PCR results but positive serology tests $(6-8,10,13)$. One had a negative RT-PCR result despite having had a positive RT-PCR result a few days earlier (12). The results of RT-PCR swab test and serology were both positive in one case (14). The two remaining patients had a positive RT-PCR test but did not have serology tests $(9,11)$. These findings suggested that MIS-A probably occurred during the post-acute phase of the disease.

All the patients presented with fever. Seven had a fever for 5 to 7 days prior to hospital admission, while two did not report fever duration. Most $(n=7)$ had digestive symptoms upon admission
(7,9-14), with the most common diarrhea $(n=6)$, followed by vomiting $(n=4)$ and bilateral enlarged parotid glands $(n=1)$. Rash $(n=4)(8-10,14)$ and neck pain $(n=3)(11-13)$ with or without lymphadenopathy were also common.

There was multi-organ effect in all cases. Involvement of the cardiovascular system was the most common $(n=7)$ $(6,7,10,12-15)$ and was documented via echocardiography in four cases. The four cases had an acute myocardial dysfunction with left ventricular systolic dysfunction and pericardial effusion. Two had ventricular fibrillation $(11,12)$ and two other a dilated inferior vena cava $(10,14)$. One of these patients also had overloaded right ventricular pressure and mild enlargement of the main pulmonary artery and hyperkinetic left ventricle (14).

The other manifestations were digestive $(n=7)(7,9-14)$, ophthalmic $(n=6)(8-11,13,14)$, renal $(n=4)(6,11,12,14)$, dermatologic $(n=5)(6,8-10,13)$, pulmonary $(n=2)(7,12)$ and neurologic $(n=1)(6)$.

C-reactive protein (CRP) test results and lymphocyte counts were reported in eight cases, and D-dimers and troponin in six cases. All cases had elevated inflammatory markers. The inflammatory markers that were most commonly elevated were $\operatorname{CRP}(n=8)(6,7,9-14)$, followed by D-dimers $(n=6)(7,9,11-14)$ and troponin $(n=6)(6-11)$. Lymphopenia was also common $(n=6)$ $(7-9,11,12,14)$. Three authors excluded rheumatic disease, HIV and hepatitis infection $(9,11,13)$.

Intravenous immunoglobulin (IVIG; $n=4)(8,9,11,14)$, prednisolone $(n=3)(8,9,13)$ and aspirin $(n=3)(7,13,14)$ were the most common treatments. Immunoglobulin was not given in one case because the patient responded well to aspirin (7). In another case, prednisolone was not provided because the patient had a concomitant tracheal aspiration positive for Klebsiella aerogenes (syn: Enterobacter aerogenes) that was then treated with trimethoprim sulfamethoxazole (6). One patient did not receive any specific treatment; she died while being evaluated for admission (12).

Of the nine patients, one died (12) and the outcome of another was not reported (9). Three patients had severe symptoms, requiring admission to the intensive care unit (ICU), but recovered $(6,7,14)$. Two patients presented with hypotension and tachycardia upon admission but did not require admission to ICU and recovered $(11,13)$. One patient presented with vasoplegic shock upon admission, had a length of stay in hospital of eight days and recovered under treatment (8). One case did not demonstrate shock-like signs and recovered under treatment (9). The case that died had been previously hospitalized for COVID-19 and discharged 12 days earlier; upon readmission she presented with rapid onset of fever and developed hemodynamic instability and ventricular fibrillation and could not be resuscitated. 


\section{Discussion}

MIS-A appears to be a rare complication of COVID-19 disease. The RT-PCR and serology results and the absence of pulmonary involvement in most cases are consistent with MIS-A occurring during the post-acute phase of COVID-19 disease.

The clinical characteristics of MIS-A share similarities with MIS-C. The pathogenesis of MIS-C involves immune dysregulation similar to Kawasaki disease, macrophage activation syndrome (MAS) and cytokine release syndrome $(16,17)$. Kawasaki disease is theorized to be from an aberrant immune response to a possible infectious trigger; it is described in children and less often in adults $(15,18)$. In the case of MIS-A, the pathogenesis is not fully understood (19). Endothelial damage seems to have led to serious complications with multi-organ involvement in the reported cases (12). This process probably occurs post-infection based on the timing of the rise of MIS-C cases and peak of COVID-19 in the communities in which these cases were found $(16,17)$.

While we identified some common features, the clinical presentations in the case reports of the MIS-A patients varied. For example, ophthalmologic signs (9) were predominant in one case and cardiac signs in another (6). Further studies are required on MIS-C pathophysiology and how it contributes to MIS-A pathogenesis.

The approach to management of children with MIS-C is evolving; management does require multidisciplinary care and a caseby-case approach. Since MIS-C is most likely a post-infectious complication rather than an active infection, the role of antivirals is not clear (20). Those that meet the criteria for Kawasaki disease may benefit from IVIG, as might those with moderate to severe MIS-C (20). Patients who may benefit from this treatment may include those with cardiac involvement or in shock states. Steroids might be considered for those who have severe or refractory shock (20). Other adjunctive therapies (IL-1 inhibitors or convalescent plasma) and their place in the treatment of MIS-C is uncertain (20). How these treatment options can be applied to MIS-A patients is also currently unknown. We need further studies outside of controlled clinical trials to ascertain the role of IVIG, steroids and other immunomodulatory agents in treating suspected cases of MIS-A (21).

\section{Limitations}

We based this current review on nine case reports from three countries. Although case reports can help in identifying new trends or diseases, there are limitations. Information from the case reports is difficult to generalize because patients have different backgrounds and are not representative of the population.
Currently, there is no case definition for MIS-A. Using the MIS-C case definition (minus age) has its challenges, as there are at least four definitions (see Appendix $\mathrm{C}$ ). In addition, how each case met the definition was not always clear. For example, authors of the case reports did not always specify how they excluded all other potential causes of the multisystem inflammatory syndrome or report the duration of fever or presence of comorbidities. There was also a lack of information about ethnicity and severity of the disease. For example, when hypotension was identified, the presence or absence of shock-like syndrome was not always specified.

These are preliminary findings; additional studies will lead to a better understanding of common epidemiologic and clinical characteristics of this condition.

\section{Conclusion}

The case descriptions revealed similarities in clinical features such as fever, digestive symptoms, cardiac involvement and elevated inflammatory markers. The RT-PCR and serology results and the absence of pulmonary involvement suggest that MIS-A occurred during the post-acute phase of COVID-19 disease. All patients were hospitalized, three required admission to the ICU and one died. The most common treatments were IVIG, prednisolone and aspirin.

The findings suggest that MIS-A is a severe complication of COVID-19 disease that can lead to death. Early recognition of MIS-A may improve outcomes. A case definition for MIS-A is needed to help standardize reporting and facilitate disease recognition. Further studies to improve our understanding of pathogenesis of MIS-A will help improve treatment decisions and prevent sequelae and death.

\section{Authors' statement}

NA - Methodology, investigation, writing-original draft

RE - Conceptualization, writing-review and editing, supervision

MS - Writing-review and editing

LW - Writing-review and editing

NB - Writing-review and editing

TD - Writing-review and editing

\section{Competing interests}

None.

\section{Acknowledgments}

We acknowledge our collaborators at the Emerging Science Group for their help in this work. 


\section{Funding}

None.

\section{References}

1. Wu Z, McGoogan JM. Characteristics of and important lessons from the coronavirus disease 2019 (COVID-19) outbreak in China: summary of a report of 72314 cases from the Chinese Center for Disease Control and Prevention. JAMA 2020;323(13):1239-42. DOI PubMed

2. Worldometer. Worldometer COVID-19 coronavirus pandemic [Internet]. (updated 2020-05-29; accessed 2020-05-29). https://www.worldometers.info/coronavirus/

3. Manabe T, Akatsu H, Kotani K, Kudo K. Trends in clinical features of novel coronavirus disease (COVID-19): A systematic review and meta-analysis of studies published from December 2019 to February 2020. Respir Investig 2020;58(5):409-18. DOI PubMed

4. Polak SB, Van Gool IC, Cohen D, von der Thüsen JH, van Paassen J. A systematic review of pathological findings in COVID-19: a pathophysiological timeline and possible mechanisms of disease progression. Mod Pathol 2020;33(11):2128-38. DOI PubMed

5. Radia T, Williams N, Agrawal P, Harman K, Weale J, Cook J, Gupta A. Multi-system inflammatory syndrome in children \& adolescents (MIS-C): a systematic review of clinical features and presentation. Paediatr Respir Rev 2020;S1526-0542(20)30117-2. DOI

6. Boudhabhay I, Rabant M, Coupry L-M, Marchal A, Lubka TR, El-Karoui K, Monchi M, Pourcine F. Adult post COVID-19 multisystem inflammatory syndrome and thrombotic microangiopathy. Preprint. Research Square; (updated 2020-09-16; accessed 2020-05-29). DOI

7. Chowdhary A, Joy E, Plein S, Abdel-Rahman SE. Multisystem inflammatory syndrome in an adult with SARS-CoV-2 infection. Eur Heart J Cardiovasc Imaging 2021;22(5):e17. DOI PubMed

8. Jones I, Bell LC, Manson JJ, Last A; UCLH COVID Response Team. An adult presentation consistent with PIMS-TS. Lancet Rheumatol 2020;2(9):e520-1. DOI PubMed

9. Lidder AK, Pandit SA, Lazzaro DR. An adult with COVID-19 kawasaki-like syndrome and ocular manifestations. Am J Ophthalmol Case Rep 2020;20:100875. DOI PubMed

10. Moghadam $P$, Blum $L$, Ahouach $B$, Radjou A, Lambert $C$, Scanvic A, Martres P, Decalf V, Bégon E, Bachmeyer C. Multisystem inflammatory syndrome with particular cutaneous lesions related to COVID-19 in a young adult. Am J Med 2021;134(1):e36-7. DOl PubMed

11. Shaigany S, Gnirke M, Guttmann A, Chong H, Meehan S, Raabe V, Louie E, Solitar B, Femia A. An adult with Kawasaki-like multisystem inflammatory syndrome associated with COVID-19. Lancet 2020;396(10246):e8-10. DOI PubMed
12. Fox SE, Lameira FS, Rinker EB, Vander Heide RS. Cardiac endotheliitis and multisystem inflammatory syndrome after COVID-19. Ann Intern Med 2020;173(12):1025-7.

DOI PubMed

13. Sokolovsky $S$, Soni $P$, Hoffman $T$, Kahn $P$, Scheers-Masters J. COVID-19 associated Kawasaki-like multisystem inflammatory disease in an adult. Am J Emerg Med 2021;39(39):253.e1-2. DOI PubMed

14. Kofman AD, Sizemore EK, Detelich JF, Albrecht B, Piantadosi AL. A young adult with COVID-19 and multisystem inflammatory syndrome in children (MIS-C)-like illness: a case report. BMC Infect Dis 2020;20(1):716. DOI PubMed

15. Stankovic K, Miailhes P, Bessis D, Ferry T, Broussolle C, Sève P. Kawasaki-like syndromes in HIV-infected adults. J Infect 2007;55(6):488-94. DOI PubMed

16. Whittaker E, Bamford A, Kenny J, Kaforou M, Jones CE, Shah P, Ramnarayan P, Fraisse A, Miller O, Davies P, Kucera F, Brierley J, McDougall M, Carter M, Tremoulet A, Shimizu C, Herberg J, Burns JC, Lyall H, Levin M; PIMS-TS Study Group and EUCLIDS and PERFORM Consortia. Clinical characteristics of 58 children with a pediatric inflammatory multisystem syndrome temporally associated with SARS-CoV-2. JAMA 2020;324(3):259-69. DOI PubMed

17. Mahase E. Covid-19: cases of inflammatory syndrome in children surge after urgent alert. BMJ 2020;369:m1990. DOI PubMed

18. Drago F, Javor S, Ciccarese G, Cozzani E, Parodi A. A case of complete adult-onset Kawasaki disease: a review of pathogenesis and classification. Dermatology 2015;231(1):5-8. DOI PubMed

19. Morris SB, Schwartz NG, Patel P, Abbo L, Beauchamps $L$, Balan S, Lee EH, Paneth-Pollak R, Geevarughese A, Lash MK, Dorsinville MS, Ballen V, Eiras DP, Newton-Cheh C, Smith E, Robinson S, Stogsdill P, Lim S, Fox SE, Richardson G, Hand J, Oliver NT, Kofman A, Bryant B, Ende Z, Datta D, Belay E, Godfred-Cato $S$. Case series of multisystem inflammatory syndrome in adults associated with SARS-CoV-2 infection United Kingdom and United States, March-August 2020. MMWR Morb Mortal Wkly Rep 2020;69(40):1450-6. DOI PubMed

20. Son MB, Friedman K. COVID-19: Multisystem inflammatory syndrome in children (MIS-C) management and outcome: features of Kawasaki disease. Alphen aan den Rijn (NL): Wolters Kluwer; (updated 2021; accessed 2021-03-25). https://www.uptodate.com/contents/covid-19-multisysteminflammatory-syndrome-in-children-mis-c-managementand-outcome?search=COVID\%2019\%20multi\%20 inflammatory\%20response\&topicRef $=128389 \&$ source $=$ see link\#H1902242396

21. Tenforde MW, Morris SB. Multisystem inflammatory syndrome in adults: coming into focus. Chest 2021;159(2):471-2. DOI PubMed 
22. World Health Organization. Multisystem inflammatory syndrome in children and adolescents temporally related to COVID-19: scientific brief. Geneva: WHO; (updated 2020-05-15; accessed 2020-12-14). https://www.who.int/news-room/commentaries/detail/ multisystem-inflammatory-syndrome-in-children-andadolescents-with-covid-19

23. Centers for Disease Control. Information for healthcare providers about multisystem inflammatory syndrome in children (MIS-C). Atlanta (GA): CDC; (updated 2020; accessed 2020-12-14). https://www.cdc.gov/ mis-c/hcp/
24. Royal College of Paediatrics and Child Health. Paediatric multisystem inflammatory syndrome temporally associated with COVID-19 (PIMS) - guidance for clinicians. London (UK): RCPCH; (updated 2020; accessed 2020-12-06). https://www. rcpch.ac.uk/resources/paediatric-multisystem-inflammatorysyndrome-temporally-associated-covid-19-pims-guidance

25. Berard RA, Tam H, Scuccimarri R, Haddad E, Morin MP, Chan KJ, Dahdah NS, McCrindle BW, Price VE, Yeung RS, Laxer RM. Acute Care Committee Paediatric inflammatory multisystem syndrome temporally associated with COVID-19. Ottawa (ON): Canadian Pediatric Society; (updated 2020-07-06; accessed 2020-12-14). https://www.cps.ca/documents/position/pims

\section{Appendices}

\section{Appendix A: Comparison of nine MIS-A cases in the COVID-19 literature published up to November 2020}

\begin{tabular}{|c|c|c|c|c|c|c|c|c|c|c|}
\hline \multicolumn{2}{|c|}{$\begin{array}{l}\text { Patient/studies } \\
\text { characteristics }\end{array}$} & $\begin{array}{c}\text { Boudhabhay } \\
\text { et al., } 2020 \\
\text { (6) }\end{array}$ & $\begin{array}{l}\text { Chowdhary } \\
\text { et al., } 2021 \\
\text { (7) }\end{array}$ & $\begin{array}{c}\text { Fox et } \\
\text { al., 2020 } \\
\text { (12) }\end{array}$ & $\begin{array}{l}\text { Jones et } \\
\text { al., } 2020 \\
\quad(8)\end{array}$ & $\begin{array}{l}\text { Kofman } \\
2020(14)\end{array}$ & $\begin{array}{l}\text { Lidder et } \\
\text { al., } \\
2020 \text { (9) }\end{array}$ & $\begin{array}{c}\text { Moghadam } \\
\text { et al., 2020 } \\
\text { (10) }\end{array}$ & $\begin{array}{c}\text { Sokolovsky } \\
\text { et al., 2020 } \\
\text { (13) }\end{array}$ & $\begin{array}{c}\text { Shaigany } \\
\text { et al., } \\
2020 \\
(11)\end{array}$ \\
\hline \multirow{4}{*}{ Background } & Ethnicity & African & NR & $\begin{array}{l}\text { African } \\
\text { American }\end{array}$ & African & NR & NR & White & Hispanic & Hispanic \\
\hline & Age, years & 46 & 26 & 31 & 21 & 25 & 45 & 21 & 36 & 45 \\
\hline & Sex & Male & Male & Female & Male & Female & Male & Male & Female & Male \\
\hline & $\begin{array}{l}\text { Presence of } \\
\text { comorbidity }\end{array}$ & $x$ & NR & $x$ & NR & - & - & NR & - & - \\
\hline \multirow{8}{*}{$\begin{array}{l}\text { Symptoms } \\
\text { and system/ } \\
\text { organ } \\
\text { involved }\end{array}$} & Fever & $x$ & $x$ & $x$ & $x$ & - & $x$ & $x$ & $\mathrm{x}$ & $x$ \\
\hline & Cardiovascular & $x$ & $x$ & $x$ & - & $x$ & $x$ & $x$ & $x$ & $x$ \\
\hline & Digestive & - & $x$ & $x$ & - & $x$ & $x$ & $x$ & $x$ & $x$ \\
\hline & Ophthalmic & - & - & - & $x$ & $x$ & $x$ & $x$ & $x$ & $x$ \\
\hline & Renal & $x$ & - & $x$ & - & $x$ & - & - & - & $x$ \\
\hline & Dermatologic & $x$ & - & - & $x$ & - & $x$ & $x$ & $x$ & - \\
\hline & Pulmonary & - & $x$ & $x$ & - & - & - & - & - & - \\
\hline & Neurologic & $x$ & - & - & - & - & - & - & - & - \\
\hline \multirow{2}{*}{$\begin{array}{l}\text { RT-PCR and } \\
\text { serology test } \\
\text { results }\end{array}$} & RT-PCR & Negative & Negative & Negative & Negative & Positive & Positive & Negative & Negative & Positive \\
\hline & Serology & Positive & Positive & NR & Positive & Positive & NR & Positive & Positive & NR \\
\hline \multirow{4}{*}{$\begin{array}{l}\text { Elevated } \\
\text { inflammatory } \\
\text { markers and } \\
\text { lymphopenia }\end{array}$} & CRP & $x$ & $x$ & $x$ & NR & $x$ & $x$ & $x$ & $x$ & $x$ \\
\hline & Troponin & $x$ & $x$ & NR & $x$ & - & $x$ & $x$ & NR & $x$ \\
\hline & D-dimers & NR & $x$ & $x$ & NR & $x$ & $x$ & NR & $x$ & $x$ \\
\hline & Lymphopenia & NR & $x$ & $x$ & $x$ & $x$ & $x$ & - & - & $x$ \\
\hline \multicolumn{2}{|c|}{$\begin{array}{l}\text { Exclusion of other infective and } \\
\text { inflammatory conditions }\end{array}$} & NR & NR & NR & $x$ & NR & $x$ & $x$ & $x$ & $x$ \\
\hline \multirow{3}{*}{ Treatment } & Immunoglobulin & - & - & - & $x$ & $x$ & $x$ & - & - & $x$ \\
\hline & Prednisolone & - & - & - & $x$ & - & $x$ & - & $x$ & - \\
\hline & Aspirin & - & $x$ & - & - & $x$ & - & - & $x$ & - \\
\hline \multicolumn{2}{|l|}{ Outcome } & Recovery & Recovery & Death & Recovery & Recovery & NR & Recovery & Recovery & Recovery \\
\hline
\end{tabular}

Abbreviations: CRP, C-reactive protein; NR, not reported; RT-PCR, reverse transcription polymerase chain reaction; -, not present characteristic; $\mathrm{X}$, reported as present 
Appendix B: Summary of case reports on multisystem inflammatory syndrome in adults (MIS-A) (n=9)

\section{Case report/}

demographic

characteristics

and past medical

$$
\text { history }
$$

Boudhabhay et al., 2020 (6)

France

16 September 2020

The patient was a 46-year-old male of African descent with a history of hypertension and obesity
MIS-A clinical and laboratory characteristics

Treatment/severity and outcome
Fever and other signs and symptoms:

- Admitted for hypertensive emergency $(189 / 123 \mathrm{mmHg})$ and fever (duration not reported)

Evidence of coagulopathy and renal involvement:

- Acute kidney injury: Serum creatinine (sCr) level was $169 \mu \mathrm{mol} / \mathrm{L}$ associated with $1 \mathrm{~g} /$ day proteinuria, aseptic pyuria, no hematuria and low natriuresis $(<20 \mathrm{mmol} / \mathrm{L})$

- Renal biopsy light microscopy revealed typical lesions of thrombotic microangiopathy (TMA) including fibrin thrombi within glomeruli and myxoid intimal alterations of arterioles and small to medium-sized renal arteries

- On Day 4, the patient presented evanescent facial erythema and developed acute myocardial dysfunction with reduced left ventricular ejection fraction to $40 \%$, pericardial effusion

- On Day 5, the patient presented with neurologic impairment. Abnormal supratentorial periventricular magnetic resonance imaging (MRI) signals responsible for a restriction of the diffusion due to an acute vasculitis

PCR and serology for SARS-CoV-2:

- RT-PCR negative, IgM negative and IgG positive (no previous COVID-19 symptoms were reported)

Inflammatory markers:

- CRP level was $312 \mathrm{mg} / \mathrm{L}$

- Thrombocytopenia: neutrophil count was $18.7 \times 10^{9} / \mathrm{L}$

- High sensitive troponin (hsTroponin) elevation
- No immunosuppressive treatment was introduced because of concomitant tracheal aspiration positive for Klebsiella aerogenes treated with trimethoprim sulfamethoxazole

- Dobutamine and renal replacement therapy (RRT)

- Specific complement inhibition with eculizumab therapy (900 mg)

- On Day 5 of hospitalization, neurologic impairment presented with coma leading to intubation and mechanical ventilation

The patient was discharged after 30 days in hospital

- Vasopressor therapy, high-dose aspirin and broad-spectrum antibiotics in intensive care

- Immunomodulatory therapy was not given due to the good response to aspirin

The patient was admitted to the ICU and recovered over 10 days. or absence of comorbidity was not reported

Exposure to SARS-CoV-2 was reported
- Patient was admitted after five days of fever

- Patient was hypotensive and hypoxic upon admission

One or more organs involved (pulmonary, cardiac, digestive):

- Initial transthoracic echocardiography demonstrated severe left ventricular systolic dysfunction with pericardial effusion

- CT of the abdomen demonstrating mesenteric lymphadenopathy and small bowel edema

PCR and serology for SARS-CoV-2:

- RT-PCR negative, lgG and IgM positive serology

Inflammatory markers:

- CRP: $419 \mathrm{mg} / \mathrm{L}$

- Ferritin: $3,275 \mathrm{lg} / \mathrm{L}$ (normal $<322 \mu \mathrm{g} / \mathrm{L}$ )

- Procalcitonin: $164 \mathrm{lg} / \mathrm{L}$ (normal $<50 \mu \mathrm{g} / \mathrm{L})$

- Troponin I: $2,030 \mathrm{ng} / \mathrm{L}$ (normal $<57 \mathrm{ng} / \mathrm{L}$ )

- D-dimer: $2,722 \mathrm{ng} / \mathrm{mL}$ (normal $<220 \mathrm{ng} / \mathrm{mL}$ ) 


\section{Appendix B: Summary of case reports on multisystem inflammatory syndrome in adults (MIS-A) ( $n=9)$ (continued)}

\section{Case report/}

demographic

characteristics

and past medical history

Fox et al., 2020 (12)

United States

July 2020

The patient was a 31-year-old African American female

Her comorbidities included hypertension treated with lisinopril, diabetes with poor adherence to metformin and glizide, and obesity (body mass index $[\mathrm{BMI}]=$ $36.1 \mathrm{~kg} / \mathrm{m}^{2}$ )

She had been discharged 12 days earlier after a hospitalization for COVID-19 disease with a positive RT-PCR

Jones et al., 2020 (8) United Kingdom

The date the study was conducted was not reported September 2020

The patient was a 21-year-old male of African descent

The presence or absence of comorbidity was not reported
MIS-A clinical and laboratory characteristics

Treatment/severity and outcome

The patient was admitted for sudden fever $39.8^{\circ} \mathrm{C}$ (duration not specified), tachycardia (120 beats/min), left-sided neck pain, nausea and vomiting

Inflammatory markers:

- D-dimer level of $2.48 \mathrm{nmol} / \mathrm{L}$ (normal $<1.37 \mathrm{nmol} / \mathrm{L}$ )

- CRP levels $165 \mathrm{mg} / \mathrm{L}$, then $580 \mathrm{mg} / \mathrm{L}$ (normal <9 mg/L)

- Ferritin level, $411.2 \mu \mathrm{g} / \mathrm{L}$ (normal $10-150 \mu \mathrm{g} / \mathrm{L}$ )

- Lactic acid level, $3.1 \mathrm{mmol} / \mathrm{L}$ (normal 0.3-2.0 mmol/L)

- Lymphopenia

One or more organs involved (pulmonary, cardiac, parotids, renal):

- CT scan of her neck showed bilaterally enlarged parotid glands and swelling in the posterior nasopharynx to oropharynx

- CT scan of her chest showed interval improvement of bibasilar ground-glass opacities, with cervical and anterior mediastinal lymphadenopathy

- Creatinine level $202.44 \mu \mathrm{mol} / \mathrm{L}(44.20-97.24 \mu \mathrm{mol} / \mathrm{L})$; glomerular filtration rate $32 \mathrm{~mL}$ $\mathrm{min} / 1.73 \mathrm{~m}^{2}$ (>89 mL/min/1.73 $\left.\mathrm{m}^{2}\right)$

PCR and serology for SARS-CoV-2:

- RT-PCR was positive 12 days prior to readmission

- MISC-A, RT-PCR was negative at readmission and serology was not performed

Fever and other signs or symptoms:

- Six days of fever

- Admitted for abdominal pain associated with constipation, anorexia

- Transient maculopapular palmar rash four days into illness

- Non-exudative conjunctivitis

- Cervical lymphadenopathy

- Cracked lips and prominent lingual papillae

PCR and serology for SARS-CoV-2:

- RT-PCR negative and serology was strongly positive, suggesting recent exposure to SARS-CoV-2

One or more organs involved:

- Rash

- Conjunctivitis

- Cervical lymphadenopathy

- Cracked lips and prominent lingual papillae

Inflammatory markers:

- Lymphopenia

- Elevated inflammatory and elevated troponin T

- Other infective and inflammatory conditions were excluded
- IVIG

- Methylprednisolone

The patient was

discharged after a length of hospital stay of eight days. 


\section{Appendix B: Summary of case reports on multisystem inflammatory syndrome in adults (MIS-A) ( $\mathrm{n}=9$ ) (continued)}

\section{Case report/}

demographic

characteristics

and past medical history

Kofman et al., 2020

(14)

United States

The date the study was conducted was not reported

September 2020

The patient was a 25-year-old female; her ethnicity was not reported

She was a nonsmoker, did not use drugs, was not taking any prescription medications and had no known allergies

She had taken ibuprofen and acetaminophen over the previous week for symptom relief

Lidder et al., 2020 (9)

\section{United States}

May 2020

The case was a 45-year-old male with no comorbidities

Ethnicity was not reported

\section{MIS-A clinical and laboratory characteristics}

Treatment/severity and outcome

Fever and other signs and symptoms:

- One week of low grade fever, weakness, dyspnea, fatigue

- Also developed mild cough, sore throat, vomiting, diarrhea and lymph node swelling

Upon admission:

- She was afebrile, with mild hypotension (blood pressure $98 / 56 \mathrm{mmHg}$ )

- Oxygen saturation was normal on room air

- She appeared ill, with tender cervical lymphadenopathy

- Significant conjunctival injection without perilimbal sparing; injected, erythematous and cracked lips

- Tenderness to palpation in the left lower abdominal quadrant

One or more organs involved (renal, cardiac, digestive, ocular):

- Acute kidney injury: Creatinine $7.74 \mathrm{mg} / \mathrm{dL}$ (normal: $0.5-1.2 \mathrm{mg} / \mathrm{dL}$ ) and leukocytosis

- Point-of-care echocardiogram revealed a dilated inferior vena cava and overloaded right ventricular pressure

- CT angiogram of the chest showed mild enlargement of the main pulmonary artery

- CT abdomen/pelvis demonstrated mild peripancreatic fat stranding, felt to possibly represent acute uncomplicated pancreatitis, as well as nonspecific bilateral perinephric fat stranding

- Conjunctivitis

PCR and serology for SARS-CoV-2:

- Positive RT-PCR and IgG serology

Inflammatory markers:

- CRP: $90 \mathrm{mg} / \mathrm{L}$ (normal: 0-10 mg/L)

- D-dimer: $960 \mathrm{mg} / \mathrm{L}$ (normal: 0-574 mg/L)

- Ferritin: $798 \mathrm{ng} / \mathrm{ml}$ (normal: $11-307 \mathrm{ng} / \mathrm{mL}$ )

- Lymphocytes: 3\% (normal: 19-53)

Fever and other signs and symptoms:

- Fever for five days, sore throat, diarrhea, eye redness, eyelid swelling and a diffuse rash including bilateral upper and lower eyelids

One or more organs involved (renal, cardiac, digestive, ophthalmologic):

- A transthoracic echocardiogram demonstrated global hypokinesis and a reduced ejection fraction of $40 \%$

- CT imaging showed unilateral cervical lymphadenopathy with a lymph node measuring $1.8 \mathrm{~cm}$

- Photophobia and swollen eyelids; no vision changes including blurry vision and eye pain

- Uncorrected near visual acuity was $20 / 20$ bilaterally

- Bilateral superficial punctate keratitis, symmetric anterior chamber inflammation with 10-15 cells per high power field, and normal intraocular pressure. Dilated fundus exam was notable only for one small peripheral cotton wool spot in each eye

- Punch biopsy of his erythema multiforme-like rash

- Showed sparse superficial perivascular infiltrate of lymphocytes with neutrophils and scattered eosinophils, suggestive of toxic shock syndrome

Excluding other cause:

- Testing for myositis and HIV was negative

- An exhaustive rheumatologic workup, including ANA, RF, anti-CCP, anti-Smith, anti-dsDNA p-ANCA/MPO, c-ANCA/PR3, was negative

- Blood cultures were negative

PCR and serology for SARS-CoV-2:

- Positive RT-PCR

Inflammatory markers:

- Lymphopenia

- Ferritin, CRP, ESR, D-dimer and troponin were elevated
- Aggressive fluid resuscitation and vasopressor

- $\quad \mathrm{VIG}, 2 \mathrm{~g} / \mathrm{kg}$ split equally between hospital days 2 and 3

- Aspirin $325 \mathrm{mg}$ daily for seven days

- Patient was offered remdesivir under an Emergency Use Authorization (EUA) basis, but declined

- At discharge she was prescribed a seven-day course of apixaban for COVID-19-associated coagulopathy per Emory University Hospital COVID-19 treatment guidelines

The patient was admitted to the ICU twice during her hospital stay. She was discharged on Day 5.

- Ophthalmic lubricating therapy in addition to prednisolone acetate $1 \%$ eye drops four times daily for his photophobia in the setting of anterior chamber inflammation

- IVIG and an interleukin-6 (IL-6) inhibitor (tocilizumab) in addition to using a topical triamcinolone ointment for his diffuse rash

The length of hospital stay was not reported, but the patient did not demonstrate shock-like signs. 


\section{Appendix B: Summary of case reports on multisystem inflammatory syndrome in adults (MIS-A) ( $n=9)$ (continued)}

\section{Case report/}

demographic

characteristics

and past medical history

Moghadam et al., 2020 (10)

France

The date the study was conducted was not reported

July 2020

21-year-old White male who did not smoke or use drugs

The presence or absence of comorbidity was not reported
MIS-A clinical and laboratory characteristics

Treatment/severity and outcome

reported

Sokolovsky et al., 2020 (13)

United States

The date the study was conducted was not reported

June 2020

The case was a 36-year-old Hispanic female with no known comorbidity
Fever and other signs and symptoms:

- Fever and non-bloody watery diarrhea lasting for seven days

- Asymptomatic rash over his trunk and palms, consisting of erythematous round-shaped macules with a darker and raised rim, $1-3 \mathrm{~cm}$ in diameter

- Bilateral conjunctivitis

- Blood pressure $80 / 40 \mathrm{mmHg}$

- Respiratory rate was 38 breaths/min, and oxygen saturation was $97 \%$ on ambient air

One or more organs involved (cardiac, digestive, pleural):

- Electrocardiogram showed diffuse negative T-waves, and echocardiography displayed hyperkinetic left ventricle with normal ejection fraction, normal right cavities and dilated non-compressible inferior vena cava

- Thoraco-abdominal CT scan showed:

- Signs of congestive heart failure

- Bilateral pleural effusion

- Wall thickening of the right colon

- Respiratory function deterioration

PCR and serology for SARS-CoV-2:

- Negative RT-PCR and lgG-positive serology

Inflammatory markers:

- Lymphocytes: $900 / \mathrm{mm}^{3}$

- CRP: $365 \mathrm{mg} / \mathrm{L}$

- Procalcitonin: $3.4 \mathrm{ng} / \mathrm{mL}$

- Ferritin: $1,282 \mathrm{mg} / \mathrm{L}$ (normal $<30$ )

- Lactate: $2.4 \mathrm{mmol} / \mathrm{L}$ (normal <1.6)

- Troponin level: 550 ng/L (normal <34)

- Cutaneous biopsy showed a slightly inflammatory infiltrate in upper dermis. Direct cutaneous immunofluorescence was negative

Exclusion of other causes:

- Extensive infectious inquiry and search for antinuclear antibodies were negative

- The rash was particular and diagnosis of erythema multiforma and subacute lupus erythematosus were ruled out

Fever and other signs and symptoms:

- One week of fever, abdominal pain, vomiting and diarrhea

- Two days of a diffuse rash and arthralgias

- Tachycardia, tachypnea, hypotensive

- Classic phenotype of complete Kawasaki disease: bilateral nonexudative conjunctivitis mucositis with cracked lips, edema of the bilateral hands and feet, diffuse maculopapular rash and cervical lymphadenopathy

One or more organs involved (cardiac, digestive):

- CT angiogram of the chest: normal lung parenchyma and a trace right pleural effusion

- CT abdomen/pelvis illustrated mild circumferential gallbladder wall thickening and a small area of colitis

- Echocardiogram after treatment with IVIG revealed an ejection fraction of $65 \%$ with moderate tricuspid valve regurgitation. Subsequent coronary computed tomography angiography (CCTA) was normal except for a trace pericardial effusion

PCR and serology for SARS-CoV-2:

- Negative RT-PCR and lgG-positive serology

Inflammatory markers:

- CRP: $30 \mathrm{mg} / \mathrm{dL}$ (normal 0.0-0.9)

- D-dimer: $652 \mathrm{ng} / \mathrm{mL}$ (normal <318)

Exclusion of other cause:

- Anti-dsDNA, anti-Smith, anti-RNP, SSB, RF, CCP, ANCA, ASO and anti-Jo-1 antibodies were negative

- HIV and hepatitis panels were negative
- Volume resuscitation

- Noradrenaline

- Antibiotics (i.e. ceftriaxone and amikacin)

- High-flow nasal oxygenation

The patient stayed in the ICU for eight days and recovered.
- Fluid resuscitation for shock

- A single dose of aspirin $650 \mathrm{mg}$

- IVIG $2 \mathrm{~g} / \mathrm{kg}$

- Methylprednisolone $2 \mathrm{mg} / \mathrm{kg}$ for five days followed by a prednisone taper

The patient stayed at least six days in hospital and recovered. 


\section{Appendix B: Summary of case reports on multisystem inflammatory syndrome in adults (MIS-A) ( $\mathrm{n}=9$ ) (continued)}

Case report/

demographic

characteristics

and past medical history

Shaigany et al., 2020

(11)

United States

The date the study was conducted was not reported

July 2020

The case was a 45-year-old Hispanic male

He had no known comorbidity

\section{MIS-A clinical and laboratory characteristics}

Treatment/severity and outcome

Fever and other signs and symptoms:

- Six days of fever, sore throat, diarrhea, bilateral lower extremity pain, conjunctivitis and diffuse exanthema

- Exposure to SARS-CoV-2 infection two weeks earlier

- Respiratory rate was 25-33 breaths per min

- Hypotension (systolic blood pressure $80-90 \mathrm{mmHg}$ )

- Tachycardia with episodes of atrial fibrillation with rapid ventricular response

- Bilateral, nonexudative conjunctival injection

- Tender left neck swelling with palpable lymphadenopathy, periorbital edema with overlying erythema, lip cheilitis and targetoid erythematous papules and plaques with central duskiness involving the back, palms, neck, scalp, anterior trunk and upper thighs

One or more organs involved (renal, cardiac, digestive, ophthalmologic):

- CT of the neck revealed inflammation and edema involving the bilateral lower eyelid and pre-septal space, as well as sub-occipital reactive lymphadenopathy

- Electrocardiogram demonstrated:

- ST elevations in the anterolateral leads

- Global hypokinesis of the left ventricular wall with a mild to moderately reduced ejection fraction of $40 \%$

- Diffuse conjunctivitis with chemosis as well as the presence of inflammatory cells within the anterior chamber, indicative of uveitis

- A 4-mm punch biopsy of the skin was performed on a papule on the back, with histology revealing rare intraepithelial collections of neutrophils with necrotic keratinocytes and a sparse interstitial, mixed-cell dermal infiltrate with vacuolar interface changes

PCR and serology for SARS-CoV-2:

- Positive RT-PCR

Inflammatory markers:

- Lymphopenia (0-700 lymphocytes per $\mu \mathrm{L}$ )

- ESR of $120 \mathrm{~mm} / \mathrm{hour}$

- Ferritin of $21,196 \mathrm{ng} / \mathrm{mL}$

- CRP of $546.7 \mathrm{mg} / \mathrm{L}$

- D-dimer of $2,977 \mathrm{ng} / \mathrm{mL}$

- Procalcitonin of $31.79 \mathrm{ng} / \mathrm{mL}$

- Interleukin-6 (IL-6) $117 \mathrm{pg} / \mathrm{mL}$

- Troponin $8.05 \mathrm{~g} / \mathrm{mL}$

Exclusion of other causes:

- HIV-1 and HIV-2 antibodies were negative

- Bacterial blood cultures were negative

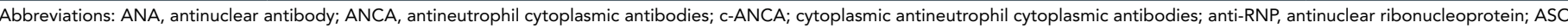

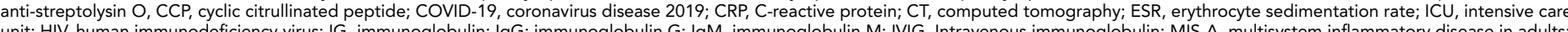

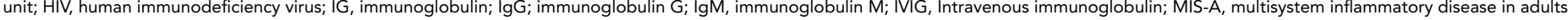

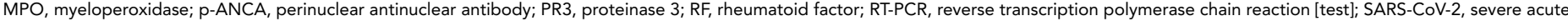
respiratory syndrome coronavirus 2 ; SSB, Sjögren's syndrome type B 


\section{Appendix C: Definitions of multisystem inflammatory syndrome in children}

\begin{tabular}{|c|c|}
\hline Authors & Definitions of MIS-C \\
\hline $\begin{array}{l}\text { World Health } \\
\text { Organization (WHO) } \\
(22)\end{array}$ & $\begin{array}{l}\text { Diagnosis of MIS-C in children and adolescents aged less than } 19 \text { years includes a positive COVID-19 test or likely } \\
\text { contact with COVID-19-positive individuals and several signs and symptoms. These include fever lasting for more than } \\
\text { three days and two of the following: } \\
\text { - Rash } \\
\text { - Bilateral non-purulent conjunctivitis } \\
\text { - Signs of muco-cutaneous inflammation (in the mouth or on the hands or feet) } \\
\text { - Hypotension or shock } \\
\text { - Myocardial dysfunction, pericarditis, valvulitis or coronary abnormalities (including echocardiogram findings or elevated } \\
\text { - troponin/NT-proBNP) } \\
\text { - Acuate gastrointestinal problems (diarrhea, vomiting or abdominal pain) } \\
\text { There must be laboratory evidence of inflammation, such as an elevated erythrocyte sedimentation rate (ESR), CRP } \\
\text { or procalcitonin. Other obvious microbial causes of inflammation such as bacterial sepsis and staphylococcal or } \\
\text { streptococcal shock syndromes must be excluded as a plausible diagnosis. }\end{array}$ \\
\hline $\begin{array}{l}\text { Centers for Disease } \\
\text { Control (CDC) (23) }\end{array}$ & $\begin{array}{l}\text { An individual below the age of } 21 \text { years presenting with fever lasting for more than } 24 \text { hours and laboratory evidence of } \\
\text { inflammation, such as an elevated CRP, ESR, fibrinogen, procalcitonin, D-dimer, ferritin, lactic acid dehydrogenase (LDH) } \\
\text { or interleukin-6, elevated neutrophils, reduced lymphocytes and low albumin. The patient must also have an evidence } \\
\text { of clinically severe illness requiring hospitalization, with multisystem organ involvement and no alternative plausible } \\
\text { diagnoses. The patient must be positive for current or recent SARS-CoV-2 infection by RT-PCR, serology or antigen test; } \\
\text { or must have been exposed to a suspected or confirmed COVID-19 case within the four weeks prior to the onset of } \\
\text { symptoms. }\end{array}$ \\
\hline $\begin{array}{l}\text { Royal College of } \\
\text { Paediatrics and Child } \\
\text { Health (RCPCH) (24) }\end{array}$ & $\begin{array}{l}\text { A child presenting with persistent fever, inflammation (neutrophilia, elevated CRP and lymphopenia) and evidence } \\
\text { of single or multi-organ dysfunction (shock, cardiac, respiratory, renal, gastrointestinal or neurologic disorder) with } \\
\text { persistent fever over } 38.5^{\circ} \mathrm{C} \text { most of the time, oxygen requirement, hypotension and other features. The laboratory } \\
\text { tests must show abnormal fibrinogen, absence of potential causative organisms (other than SARS-CoV-2), high CRP, } \\
\text { high D-dimers, high ferritin, hypoalbuminemia and/or lymphopenia. This may include children fulfilling full or partial } \\
\text { criteria for Kawasaki disease. Any other microbial cause, including bacterial sepsis, staphylococcal or streptococcal shock } \\
\text { syndromes, infections associated with myocarditis such as enterovirus must be excluded. The SARS-CoV-2 PCR testing } \\
\text { may be positive or negative. }\end{array}$ \\
\hline $\begin{array}{l}\text { Canadian Pediatric } \\
\text { Society (CPS) (25) }\end{array}$ & $\begin{array}{l}\text { The presence of high and persistent fever ( } \geq 3 \text { days) unexplained by other causes. Fever together with laboratory } \\
\text { evidence of marked systemic inflammation and temporal association with COVID-19 having been present in the } \\
\text { community should raise the index of suspicion for MIS-C. The clinical presentations described to date have included } \\
\text { fever with hyperinflammation; a Kawasaki-like syndrome; and shock or toxic shock-like states, with signs of hypotension } \\
\text { and poor perfusion related to severe myocardial dysfunction. Gastrointestinal distress, that may or may not occur with } \\
\text { neurologic signs such as neck stiffness, altered mental status or lethargy. }\end{array}$ \\
\hline
\end{tabular}

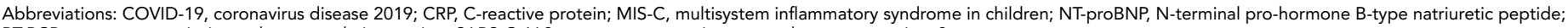
RT-PCR, reverse transcription polymerase chain reaction; SARS-CoV-2, severe acute respiratory syndrome coronavirus 2 